\title{
Finite semisimple group algebra of a normally monomial group
}

\author{
Shalini Gupta * \\ Department of Mathematics, \\ Punjabi University, Patiala, India. \\ email:shalini@pbi.ac.in \\ Sugandha Maheshwary ${ }^{\dagger}$ \\ Department of Mathematical Sciences, \\ Indian Institute of Science Education and Research, Mohali, \\ Sector 81, Mohali (Punjab)-140306, India. \\ email:sugandha@iisermohali.ac.in
}

\begin{abstract}
In this paper, the complete algebraic structure of finite semisimple group algebra of a normally monomial group is described. The main result is illustrated by computing the explicit Wedderburn decomposition of finite semisimple group algebras of various normally monomial groups. The automorphism groups of these group algebras are also determined.
\end{abstract}

Keywords : semisimple group algebra, normally monomial groups, primitive central idempotents, Wedderburn decomposition.

MSC2000 : 16S34; 20C05; 16K20

\section{Introduction}

Let $\mathbb{F}_{q}$ denote the field containing $q$ elements and let $G$ be a finite group of order relatively prime to $q$, so that the group algebra $\mathbb{F}_{q} G$ is semisimple. The knowledge of the algebraic structure of $\mathbb{F}_{q} G$ has applications in coding theory and is useful in describing the automorphism group as well as the unit group of $\mathbb{F}_{q} G$. This has attracted the attention of several authors [1, 2, 3, 8, 9, 11, 17, 18, 22.

\footnotetext{
${ }^{*}$ Corresponding author

${ }^{\dagger}$ Research supported by SERB, India (PDF/2016/000731).
} 
Broche et al. [11] gave description of semisimple group algbra $\mathbb{F}_{q} G$, when $G$ is an abelian-by-supersolvable group, by computing its primitive central idempotents and the corresponding simple components, in terms of subgroups of $G$. Basing on the work in [4] and [11], a more precise description of $\mathbb{F}_{q} G$, where $G$ is a finite metabelian group, has been given by Bakshi et al. in a series of papers [1, 2, 3].

The present paper is a contribution to the work in same series. Recall that a group $G$ is called normally monomial, if every irreducible character of $G$ is normally monomial, i.e., induced from a linear character of a normal subgroup of $G$. We provide a complete set of primitive central idempotents and the Wedderburn decomposition of $\mathbb{F}_{q} G$, when $G$ is a finite normally monomial group. Since metabelian groups are normally monomial [10], this generalises the main result of [3]. It may be remarked that normally monomial groups form a substantial class of monomial groups [7] and the rational group algebra of this class of groups has been studied [5].

The main result is given in Section 2, after setting up necessary notation and preliminaries and is illustrated on a family of metabelian groups. In Section 3, we give applications of the main result on certain $p$-groups, which include a family of non-metabelian but normally monomial groups of order $p^{7}, p$ prime, $p \geq 5$. It may be pointed out that for most of these group algebras, the GAP package Wedderga [12] practically fails to compute the Wedderburn decomposition. Further, we provide the explicit structure of $\mathbb{F}_{q} G$, for any group $G$ of order $p^{n}, p$ prime, $n<5$. For these groups, the group $\operatorname{Aut}\left(\mathbb{F}_{q} G\right)$ of $\mathbb{F}_{q^{-}}$-automorphisms of $\mathbb{F}_{q} G$ has also been computed.

\section{Notation}

Throughout the paper, $G$ denotes a finite group, and $\mathbb{F}_{q} G$ denotes the group algebra of $G$ over the field $\mathbb{F}_{q}$ containing $q$ elements, $q$ relatively prime to the order $|G|$ of $G$. The notation used are mostly standard and are listed for the ease of reader.

$$
\begin{array}{ll}
H \leq(\unlhd) G & H \text { is a subgroup (normal subgroup) of } G \\
N_{G}(H) & \text { the normalizer of } H \text { in } G, H \leq G \\
{[G: H]} & \text { the index of the subgroup } H \text { in } G, H \leq G \\
H^{g} & g^{-1} H g, g \in G, H \leq G \\
\text { core }_{G}(H) & \bigcap_{g \in G} H^{g}, \text { the largest normal subgroup of } G \text { contained in } H, H \leq G \\
& h^{-1} g^{-1} h g, g, h \in G \\
{[h, g]} & \{[h, g] \mid g, h \in G\} \\
G^{\prime} &
\end{array}
$$




$\begin{array}{ll}|X| & \text { the cardinality of the set } X \\ \langle X\rangle & \text { the subgroup generated by the subset } X \text { of } G \\ \psi^{G} & \text { the character } \psi \text { of a subgroup of } G, \text { induced to } G \\ \overline{\mathbb{F}}_{q} & \text { an algebraic closure of } \mathbb{F}_{q} \\ \operatorname{Irr}(G) & \text { the set of all the distinct irreducible characters of } G \text { over } \overline{\mathbb{F}}_{q} \\ \operatorname{ker}(\chi) & \{g \in G \mid \chi(g)=\chi(1)\}, \chi \in \operatorname{Irr}(G) \\ \mathbb{F}_{q}(\chi) & \text { the field obtained by adjoining } \chi(g), g \in G, \text { to } \mathbb{F}_{q}, \chi \in \operatorname{Irr}(G) \\ \operatorname{Gal}\left(\mathbb{F}_{q}(\chi) / \mathbb{F}_{q}\right) & \text { the Galois group of } \mathbb{F}_{q}(\chi) \text { over } \mathbb{F}_{q} \\ e(\chi) & \frac{\chi(1)}{|G|} \sum_{g \in G} \chi(g) g^{-1}, \chi \in \operatorname{Irr}(G) \\ e_{\mathbb{F}_{q}}(\chi) & \sum_{\sigma \in \text { Gal }\left(\mathbb{F}_{q}(\chi) / \mathbb{F}_{q}\right)} e(\sigma \circ \chi), \text { the primitive central idempotent of } \mathbb{F}_{q} G \\ a \mid b(a \nmid b) & \text { associated to the character } \chi, \chi \in \operatorname{Irr}(G) \\ \varphi & a \text { divides }(\text { does not divide }) b, a, b \geq 1 \\ \operatorname{gcd}(k, n) & \text { Euler's phi function } \\ S_{n} & \text { the greatest common divisor of the integers } k \text { and } n \\ \mathbb{Z}_{n} & \text { the symmetric group on } n \text { symbols, } n \geq 1 \\ \mathrm{SL}_{n}(\mathbb{F}) & \text { the cyclic group of order } n, n \geq 1 \\ H_{1} \rtimes H_{2} & \text { the group of } n \times n \text { matrices of determinant } 1, \text { over the field } \mathbb{F}, n \geq 1 \\ M_{n}(\mathbb{F}) & \text { the split extension of the group } H_{1} \text { by the group } H_{2} \\ M_{n}(\mathbb{F})^{(s)} & \text { the ring of } n \times n \text { matrices over the field } \mathbb{F}, n \geq 1 \\ & M_{n}(\mathbb{F}) \oplus M_{n}(\mathbb{F}) \oplus \ldots \oplus M_{n}(\mathbb{F}), \text { the direct sum of } s \text { copies, } s \geq 1\end{array}$

\section{$2 \quad$ Normally monomial groups}

\subsection{Strong Shoda pairs and idempotents}

For $H \unlhd K \leq G$, define

$$
\varepsilon(K, H):= \begin{cases}\hat{K}, & K=H \\ \prod(\hat{H}-\hat{L}), & \text { otherwise }\end{cases}
$$

where $\hat{K}:=\frac{1}{|K|} \sum_{k \in K} k$ and $L$ runs over the normal subgroups of $K$ which are minimal over the normal subgroups of $K$ containing $H$ properly. Set $e(G, K, H)$ to be the sum of distinct $G$-conjugates of $\varepsilon(K, H)$. A strong Shoda pair [21] of $G$ is a pair $(K, H)$ of subgroups of $G$ with the property that

(i) $H \unlhd K \unlhd N_{G}(H)$;

(ii) $K / H$ is cyclic and a maximal abelian subgroup of $N_{G}(H) / H$;

(iii) the distinct $G$-conjugates of $\varepsilon(K, H)$ are mutually orthogonal.

Two strong Shoda pairs $\left(K_{1}, H_{1}\right)$ and $\left(K_{2}, H_{2}\right)$ are said to be equivalent, if $e\left(G, K_{1}, H_{1}\right)=e\left(G, K_{2}, H_{2}\right)$ and a complete set of representatives from distinct 
equivalence classes of strong Shoda pairs of $G$ is called a complete irredundant set of strong Shoda pairs of $G$.

We recall the algorithm to compute a complete irredundant set of strong Shoda pairs of a finite normally monomial group $G$, as described in [5].

Let $\mathcal{N}$ be the set of all normal subgroups of $G$ and for $N \in \mathcal{N}$, let $A_{N}$ be a normal subgroup of $G$ containing $N$ such that $A_{N} / N$ is an abelian normal subgroup of maximal order in $G / N$. Note that the choice of $A_{N}$ is not unique. However, we need to fix one such $A_{N}$. For a fixed $A_{N}$, set

$\mathcal{D}_{N}$ : the set of all subgroups $D$ of $A_{N}$ containing $N$ such that $\operatorname{core}_{G}(D)=N$, $A_{N} / D$ is cyclic and is a maximal abelian subgroup of $N_{G}(D) / D$.

$\mathcal{T}_{N}$ : a set of representatives of $\mathcal{D}_{N}$ under the equivalence relation defined by conjugacy of subgroups in $G$.

$\mathcal{S}_{N}:\left\{\left(A_{N}, D\right) \mid D \in \mathcal{T}_{N}\right\}$.

It thus follows that if $N \in \mathcal{N}$ is such that $G / N$ is abelian, then

$$
\mathcal{S}_{N}= \begin{cases}\{(G, N)\}, & \text { if } G / N \text { is cyclic } \\ \emptyset, & \text { otherwise }\end{cases}
$$

Observe that every pair $(A, D) \in \mathcal{S}(G)$, where

$$
\mathcal{S}(G):=\bigcup_{N \in \mathcal{N}} \mathcal{S}_{N}
$$

is a strong Shoda pair of $G$. It has been proved ([5], Corollary 1) that $\mathcal{S}(G)$ is a complete irredundant set of strong Shoda pairs of $G$, if $G$ is a finite normally monomial group.

Remark 1. A crucial observation in the above algorithm to compute $\mathcal{S}(G)$, for a given finite group $G$, is that the choice of $A_{N}$ is irrelevant. For $N \in \mathcal{N}$, let $A_{N}^{(1)}$ be another normal subgroup of $G$ containing $N$ such that $A_{N}^{(1)} / N$ is an abelian normal subgroup of maximal order in $G / N$ and let $\mathcal{D}_{N}^{(1)}, \mathcal{T}_{N}^{(1)}$ and $\mathcal{S}_{N}^{(1)}$ be defined corresponding to $A_{N}^{(1)}$. Then, any pair in $\mathcal{S}_{N}^{(1)}$ is equivalent to a pair in $\mathcal{S}_{N}$ and vice-versa. This is because, if $\left(A_{N}^{(1)}, D^{(1)}\right) \in \mathcal{S}_{N}^{(1)}$ and $\psi$ is a complex linear character of $A_{N}^{(1)}$ with kernel $D^{(1)}$, then $\psi^{G}$ is irreducible and hence by ([5], Lemma 1 ), there exists $\left(A_{N}, D\right) \in \mathcal{S}_{N}$ such that $e_{\mathbb{Q}}\left(\psi^{G}\right)$, the primitive central idempotent of the rational group algebra $\mathbb{Q} G$, associated to $\psi^{G}$, is given by $e\left(G, A_{N}, D\right)$. However, in view of $\left([21]\right.$, Theorem 2.1), $e_{\mathbb{Q}}\left(\psi^{G}\right)=e\left(G, A_{N}^{(1)}, D^{(1)}\right)$. This gives that $\left(A_{N}, D\right)$ is equivalent to $\left(A_{N}^{(1)}, D^{(1)}\right)$. The reverse conclusion holds similarly. 
For a strong Shoda pair $(K, H)$ of $G$, let $\mathcal{C}(K / H)$ denote the set of $q$-cyclotomic cosets of $\operatorname{Irr}(K / H)$ containing its generators, i.e., if $\chi$ is a generator of $\operatorname{Irr}(K / H)$, then an element $C$ of $\mathcal{C}(K / H)$ containing $\chi$ is the set $\left\{\chi, \chi^{q}, \ldots, \chi^{q^{o-1}}\right\}$, where $n=|K / H|$ and $o=o_{n}(q)$, the order of $q$ modulo $n$. Suppose that $N_{G}(H)$ acts on $\mathcal{C}(K / H)$ by conjugation, i.e., for $g \in N_{G}(H), C \in \mathcal{C}(K / H)$ and $\chi \in C$, we define $C^{g}=\left\{\chi^{g}, \chi^{g q}, \ldots, \chi^{g q^{o-1}}\right\}$, where $\chi^{g}(k)=\chi\left(g k g^{-1}\right)$. Let $\mathcal{R}(K / H)$ denote the set of distinct orbits of $\mathcal{C}(K / H)$ and let $E_{G}(K / H)$ be the stabilizer of any $C \in \mathcal{C}(K / H)$ under the above action. It is easy to see that $|\mathcal{R}(K / H)|=\frac{\phi(n)\left|E_{G}(K / H)\right|}{\left|N_{G}(H)\right| o_{n}(q)}$.

For $C \in \mathcal{C}(K / H)$ and $\chi \in C$, set $\varepsilon_{C}(K, H)=\frac{1}{|K|} \sum_{g \in K}(\operatorname{tr}(\chi(\bar{g}))) g^{-1}$, where $\bar{g}$ denotes the image of $g$ in $K / H, \zeta_{n}$ a primitive $n^{\text {th }}$ root of unity in $\overline{\mathbb{F}}_{q}$ and $\operatorname{tr}:=t r_{\mathbb{F}_{q}\left(\zeta_{n}\right) / \mathbb{F}_{q}}$ denotes the trace of the field extension $\mathbb{F}_{q}\left(\zeta_{n}\right) / \mathbb{F}_{q}$. Let $e_{C}(G, K, H)$ denote the sum of distinct $G$-conjugates of $\varepsilon_{C}(K, H)$. Broche and Rio [11] proved that

$$
e_{C}(G, K, H) \text { is a primitive central idempotent of } \mathbb{F}_{q} G
$$

and

$$
\mathbb{F}_{q} G e_{C}(G, K, H) \cong M_{[G: K]}\left(\mathbb{F}_{q^{o /[E: K]}}\right),
$$

where $E=E_{G}(K / H)$ and $o=o_{[K: H]}(q)$.

\subsection{Main theorem}

The following theorem gives a complete set of primitive central idempotents of finite semisimple group algebra $\mathbb{F}_{q} G$, when $G$ is a normally monomial group. Consequently, the complete algebraic structure of $\mathbb{F}_{q} G$ is obtained.

Theorem 1 Let $\mathbb{F}_{q}$ be a finite field with $q$ elements and let $G$ be a finite group. Suppose that $\operatorname{gcd}(q,|G|)=1$. Then,

$$
E:=\left\{e_{C}(G, A, D) \mid(A, D) \in \mathcal{S}(G), C \in \mathcal{R}(A / D)\right\}
$$

is a complete set of primitive central idempotents of $\mathbb{F}_{q} G$ if, and only if, $G$ is normally monomial.

In order to prove the above theorem, we need the following lemmas:

Lemma 1 ([2], Lemma 1) Let $\mathbb{F}_{q}$ be a finite field with $q$ elements and let $G$ be a finite group. Suppose that $\operatorname{gcd}(q,|G|)=1$. Let $\psi$ be a linear character of a normal subgroup $A$ of $G$ with kernel $D$. If $\psi^{G} \in \operatorname{Irr}(G)$, then $e_{\mathbb{F}_{q}}\left(\psi^{G}\right)=e_{C}(G, A, D)$, for some $C \in \mathcal{C}(A / D)$. 
Lemma 2 ([16], Lemma 2.2) Let $A$ be an abelian normal subgroup of maximal order in a group $G$. If $\chi$ is a faithful irreducible normally monomial character of $G$, then $\chi$ is induced from $A$.

Proof. By assumption, $\chi=\beta^{G}$ for some linear character $\beta$ of a normal subgroup $B$ of $G$. As $B^{\prime} \subseteq \operatorname{core}_{G}(\operatorname{ker}(\beta))=\operatorname{ker}(\chi)=\langle 1\rangle$, we get that $B$ is abelian and hence $\chi(1)=[G: B] \geq[G: A]$. Let $\alpha$ be an irreducible constituent of $\chi_{A}$, where $\chi_{A}$ denotes the restriction of $\chi$ to $A$. By Frobenius reciprocity ([19], Corollary 4.2.2), $\chi$ is a constituent of $\alpha^{G}$, so that $\chi(1) \leq \alpha^{G}(1)=[G: A]$. Thus, $\chi(1)=[G: A]=$ $\alpha^{G}(1)$ and $\chi=\alpha^{G}$.

Proof of Theorem 1. Assume first that $E$ is a complete set of primitive central idempotents of $\mathbb{F}_{q} G$. Let $\chi \in \operatorname{Irr}(G)$, so that $e_{\mathbb{F}_{q}}(\chi)=e_{C}(G, A, D)$, where $(A, D) \in \mathcal{S}(G)$ and $C \in \mathcal{R}(A / D)$. But then, it follows from Lemma 1 that $e_{C}(G, A, D)=e_{\mathbb{F}_{q}}\left(\psi^{G}\right)$, for some linear character $\psi$ of $A$ with kernel $D$. Hence $e_{\mathbb{F}_{q}}(\chi)=e_{\mathbb{F}_{q}}\left(\psi^{G}\right)$, which implies that $\chi=\sigma \circ \psi^{G}$, where $\sigma \in \operatorname{Gal}\left(\mathbb{F}_{q}\left(\psi^{G}\right) / \mathbb{F}_{q}\right)$, i.e., $\chi$ is induced from a linear character $\sigma \circ \psi$ of a normal subgroup $A$ of $G$. Thus, $G$ is normally monomial.

Conversely, if $G$ is a normally monomial group, then for $\chi \in \operatorname{Irr}(G), \chi=\psi^{G}$, where $\psi$ is a linear character of some normal subgroup $K$ of $G$ with kernel $H$. Now, $\operatorname{ker}(\chi)=\operatorname{core}_{G}(H)=N$ (say). Let $\bar{\chi}$ be the corresponding character of $G / N$. It follows from Lemma 2 that

$$
\bar{\chi}=\bar{\eta}^{G / N}
$$

for some linear character $\bar{\eta}$ of $A_{N} / N$, where $A_{N} / N$ is an abelian normal subgroup of maximal order in $G / N$. Let $\operatorname{ker}(\bar{\eta})=L / N$ and let $\eta: A_{N} \rightarrow \overline{\mathbb{F}}_{q}$ be given by $\eta(g)=\bar{\eta}(g N), g \in A_{N}$. We have, by Eq. (2.5),$\chi=\eta^{G}$, and therefore, by Lemma 1, it follows that $e_{\mathbb{F}_{q}}(\chi)=e_{\mathbb{F}_{q}}\left(\eta^{G}\right)=e_{C}\left(G, A_{N}, L\right), C \in \mathcal{C}\left(A_{N} / L\right)$. Now, if $D \in \mathcal{T}_{N}$ is a representative of conjugacy class of $L$, then $D=L^{x}$, for some $x \in G$ and $e_{C}\left(G, A_{N}, L\right)=e_{C^{x}}\left(G, A_{N}, L^{x}\right)$, with $\left(A_{N}, D\right) \in \mathcal{S}(G)$. Consequently, $e_{\mathbb{F}_{q}}(\chi) \in E$. Moreover, it is easy to check that all elements in $E$ are distinct and hence, $E$ is a complete set of primitive central idempotents of $\mathbb{F}_{q} G$.

It thus follows from Theorem 1 and Eq. (2.4) that if $G$ is normally monomial, then

$$
\mathbb{F}_{q} G \cong \bigoplus_{(A, D) \in \mathcal{S}(G)} M_{[G: A]}\left(\mathbb{F}_{q^{o /[E: A]}}\right)^{(|\mathcal{R}(A / D)|)}
$$

where $E=E_{G}(A / D)$ and $o=o_{[A: D]}(q)$. 


\subsection{An illustration}

We first illustrate the results of Theorem 1 on a family of metabelian groups of order $2 s p^{2}, p$ an odd prime and $s \geq 1$. The structure of the rational group algebra of this family has been described in [5]. We undertake the case of finite semisimple group algebra for these groups.

Theorem 2 Let $G$ be a group generated by $a, b, x, y$ satisfying $a^{p}=b^{p}=x^{s}=$ $y^{2}=1,[a, b]=[x, y]=1, x^{-1} a x=a^{r}, x^{-1} b x=b^{r}, y^{-1} a y=b, y^{-1} b y=a$, where $p$ is an odd prime, $r$ is a positive integer such that $\operatorname{gcd}(r, p)=1$ and $s(>1)$ is the order $o_{p}(r)$ of $r$ modulo $p$. The Wedderburn decomposition of $\mathbb{F}_{q} G$ is given as follows:

(i) If $s$ is odd, then

$\mathbb{F}_{q} G \cong\left\{\begin{array}{ll}\bigoplus_{d \mid s} \mathbb{F}_{q^{o} d}^{\left(\frac{2 \phi(d)}{o}\right)} \bigoplus M_{s}\left(\mathbb{F}_{q} \frac{f g}{s}\right)^{\left(\frac{2(p-1)}{f g}\right)} \bigoplus M_{2 s}\left(\mathbb{F}_{q}^{\frac{f g}{s}}\right)^{\left(\frac{p^{2}-p}{2 f g}\right)}, & 2 \nmid f \\ \bigoplus_{d \mid s} \mathbb{F}_{\left.q^{\left(\frac{2 \phi(d)}{o}\right.}\right)}^{o_{d}} \bigoplus M_{s}\left(\mathbb{F}_{q} \frac{f g}{s}\right)^{\left(\frac{2(p-1)}{f g}\right)} \bigoplus M_{2 s}\left(\mathbb{F}_{q^{\frac{f g}{2 s}}}\right)^{\left(\frac{p-1}{f g}\right)} \bigoplus M_{2 s}\left(\mathbb{F}_{q^{\frac{f g}{s}}}\right)^{\left(\frac{(p-1)^{2}}{2 f g}\right)}, & 2 \mid f\end{array} ;\right.$

(ii) If $s$ is even, then

$\mathbb{F}_{q} G \cong\left\{\begin{array}{ll}\bigoplus_{d \mid s} \mathbb{F}_{q^{o} d}^{\left(\frac{2 \phi(d)}{o} d\right)} \bigoplus M_{s}\left(\mathbb{F}_{q} \frac{f g}{s}\right)^{\left(\frac{2(p-1)}{f g}\right)} \bigoplus M_{2 s}\left(\mathbb{F}_{q \frac{f g}{s}}\right)^{\left(\frac{(p-1)^{2}}{2 f g}\right)} \bigoplus M_{s}\left(\mathbb{F}_{q} \frac{2 f g^{\prime}}{s}\right)^{\left(\frac{(p-1)}{f g^{\prime}}\right)}, & 2 \nmid f \\ \bigoplus_{d \mid s} \mathbb{F}_{q^{o} d}^{\left(\frac{2 \phi(d)}{o}\right)} \bigoplus M_{s}\left(\mathbb{F}_{q} \frac{f g}{s}\right)^{\left(\frac{4(p-1)}{f g}\right)} \bigoplus M_{2 s}\left(\mathbb{F}_{q} \frac{f g}{s}\right)^{\left(\frac{(p-1)^{2}}{2 f g}\right)}, & 2 \mid f\end{array}\right.$,

where $o_{d}=o_{d}(q), f=o_{p}(q)=o_{2 p}(q), g=\operatorname{gcd}\left\{d\left|s: \frac{s}{d}\right| f\right\}$ and $g^{\prime}=\operatorname{gcd}\left\{d \mid \frac{s}{2}: \frac{s}{2 d}\right.$ is odd and, $\left.\frac{s}{2 d} \mid f\right\}$.

Proof. The distinct normal subgroups of $G\left([5]\right.$, Lemma 2) are $N_{0}:=\langle 1\rangle$, $N_{1}:=\langle a b\rangle, \quad N_{2}:=\left\langle a b^{-1}\right\rangle, \quad N_{3}:=\left\langle a b^{-1}, y\right\rangle, G_{d}:=\left\langle a, b, x^{d}\right\rangle, H_{d}:=\left\langle a, b, x^{d}, y\right\rangle, d \mid s$, if $s$ is odd; and, in addition, $N_{4}:=\left\langle a b, x^{\frac{s}{2}} y\right\rangle, K_{d}:=\left\langle a, b, x^{\frac{d}{2}} y\right\rangle$, with $d$ even and $d \mid s$, if $s$ is even. Further, for each normal subgroup $N$ of $G$, the corresponding set $\mathcal{S}_{N}$ of strong Shoda pairs of $G([5]$, Lemma 3$)$ is as follows:

(i) $\mathcal{S}_{N_{0}}=\{(\langle a, b\rangle,\langle b\rangle)\} \cup\left\{\left(\langle a, b\rangle,\left\langle b a^{\lambda^{i}}\right\rangle\right) \mid 1 \leq i \leq \frac{p-3}{2}\right\}$, where $\lambda$ is generator of the multiplicative group of reduced residue classes modulo $p$.

(ii) $\mathcal{S}_{N_{1}}= \begin{cases}\left\{\left(G_{s}, N_{1}\right)\right\}, & s \text { odd } \\ \left\{\left(K_{s}, N_{1}\right)\right\}, & s \text { even. }\end{cases}$

(iii) $\mathcal{S}_{N_{i}}=\left\{\left(H_{s}, N_{i}\right)\right\}, i=2,3$. 
(iv) $\mathcal{S}_{N_{4}}=\left\{\left(K_{s}, N_{4}\right)\right\}$.

(v) $\mathcal{S}_{G_{d}}= \begin{cases}\left\{\left(G, G_{d}\right)\right\}, & d \text { odd } \\ \emptyset, & d \text { even. }\end{cases}$

(vi) $\mathcal{S}_{H_{d}}=\left\{\left(G, H_{d}\right)\right\}, d \mid s$.

(vii) $\mathcal{S}_{K_{d}}=\left\{\left(G, K_{d}\right)\right\}, d \mid s, d$ even.

We tabulate the computations of parameters involved in applying Theorem 1 .

\section{$s$ odd}

\begin{tabular}{|l|c|c|c|}
\hline$\left(A_{N}, D\right) \in \mathcal{S}(G)$ & $E_{G}\left(A_{N}, D\right)$ & $o\left(A_{N}, D\right)$ & $\left|\mathcal{R}\left(A_{N} / D\right)\right|$ \\
\hline$\left(G, G_{d}\right), d \mid s$ & $G$ & $o_{2 d}(q)=o_{d}(q)$ & $\frac{\phi(d)}{o_{d}(q)}$ \\
\hline$\left(G, H_{d}\right), d \mid s$ & $G$ & $o_{d}(q)$ & $\frac{\phi(d)}{o_{d}(q)}$ \\
\hline$\left(G_{s}, N_{1}\right)$ & $\begin{array}{l}<a, b, x^{g}, y>, 2 \mid f \\
<a, b, x^{g}>, 2 \nmid f\end{array}$ & $\begin{array}{l}\frac{f g}{2 s}, 2 \mid f \\
\frac{f g}{s}, 2 \nmid f\end{array}$ & $\begin{array}{l}\frac{p-1}{f g}, 2 \mid f \\
\frac{p-1}{2 f g}, 2 \nmid f\end{array}$ \\
\hline$\left(H_{s}, N_{2}\right)$ & $<a, b, x^{g}, y>$ & $\frac{f g}{s}$ & $\frac{p-1}{f g}$ \\
\hline$\left(H_{s}, N_{3}\right)$ & $<a, b, x^{g}, y>$ & $\frac{f g}{s}$ & $\frac{p-1}{f g}$ \\
\hline$(<a, b>,<b>)$ & $<a, b, x^{g}>$ & $\frac{f g}{s}$ & $\frac{p-1}{f g}$ \\
\hline $\begin{array}{l}\left(<a, b>,<b a^{\lambda^{i}}>\right) \\
1 \leq i \leq \frac{p-3}{2}\end{array}$ & $<a, b, x^{g}>$ & $\frac{f g}{s}$ & $\frac{p-1}{f g}$ \\
\hline
\end{tabular}

\section{$s$ even}

\begin{tabular}{|l|c|c|c|}
\hline$\left(A_{N}, D\right)$ & $E_{G}\left(A_{N}, D\right)$ & $o\left(A_{N}, D\right)$ & $\left|\mathcal{R}\left(A_{N} / D\right)\right|$ \\
\hline$\left(G, G_{d}\right), d \mid s d$ odd & $G$ & $o_{2 d}(q)=o_{d}(q)$ & $\frac{\phi(d)}{o_{d}(q)}$ \\
\hline$\left(G, K_{d}\right), d \mid s, d$ even & $G$ & $o_{d}(q)$ & $\frac{\phi(d)}{o_{d}(q)}$ \\
\hline$\left(K_{s}, N_{1}\right)$ & $\left\{\begin{array}{c}<a, b, x^{g}, y>, 2 \mid f \\
<a, b, x^{g^{\prime}} y>, 2 \nmid f\end{array}\right.$ & $\left\{\begin{array}{c}\frac{f g}{s}, 2 \mid f \\
\frac{2 f g^{\prime}}{s}, 2 \nmid f\end{array}\right.$ & $\left\{\begin{array}{l}\frac{p-1}{f g}, 2 \mid f \\
\frac{p-1}{2 f g^{\prime}}, 2 \nmid f\end{array}\right.$ \\
\hline$\left(K_{s}, N_{4}\right)$ & $\left\{\begin{array}{l}<a, b, x^{g}, y>, 2 \mid f \\
<a, b, x^{g^{\prime}} y>, 2 \nmid f\end{array}\right.$ & $\left\{\begin{array}{c}\frac{f g}{s}, 2 \mid f \\
\frac{2 f g^{\prime}}{s}, 2 \nmid f\end{array}\right.$ & $\left\{\begin{array}{c}\frac{p-1}{f g}, 2 \mid f \\
\frac{p-1}{2 f g^{\prime}}, 2 \nmid f\end{array}\right.$ \\
\hline$\left(H_{s}, N_{2},\right)$ & $<a, b, x^{g}, y>$ & $\frac{f g}{s}$ & $\frac{p-1}{f g}$ \\
\hline$\left(H_{s}, N_{3}\right)$ & $<a, b, x^{g}, y>$ & $\frac{f g}{s}$ & $\frac{p-1}{f g}$ \\
\hline$(<a, b>,<b>)$ & $<a, b, x^{g}>$ & $\frac{f g}{s}$ & $\frac{p-1}{f g}$ \\
\hline $\begin{array}{l}\left(<a, b>,<b a^{\lambda^{i}}>\right) \\
1 \leq i \leq \frac{p-3}{2}\end{array}$ & $<a, b, x^{g}>$ & $\frac{f g}{s}$ & $\frac{p-1}{f g}$ \\
\hline
\end{tabular}

Consequently, Theorem 2 follows using Eq. (2.6) 


\section{Applications on p-groups}

\subsection{A family of normally monomial groups of order $p^{7}$}

We now illustrate the main result of this paper on a normally monomial group of order $p^{7}, p$ a prime, $p \geq 5$, which is not metabelian.

Theorem 3 Let $p \geq 5$ be a prime and let $G$ be the group generated by $a, b, c, d, s, r$ with the following defining relations:

$$
\begin{aligned}
& a^{p}=b^{p}=c^{p}=d^{p}=s^{p}=r^{p}=1, \\
& {[a, c]=[b, c]=[a, d]=[b, d]=1,[a, b]=[c, d],} \\
& s^{-1} a s=a, s^{-1} b s=b c, s^{-1} c s=a c d, s^{-1} d s=a d, \\
& {[s, r]=[a, b],[r, a]=[r, b]=[r, c]=[r, d]=1 .}
\end{aligned}
$$

Then,

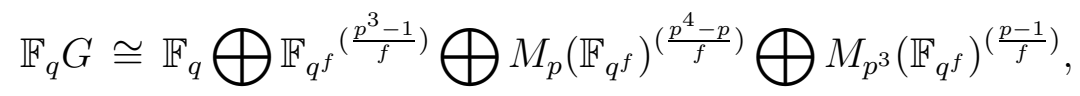

where $f=o_{p}(q)$.

Proof. Let $G$ be as in statement of the theorem. How [16] proved that $G$ is a normally monomial group, which is not metabelian. We find $\mathcal{S}(G)$ to write the Wedderburn decomposition of $\mathbb{F}_{q} G$.

First of all, we compute the set $\mathcal{N}$ of normal subgroups of $G$. For this, we begin by observing that if $\langle 1\rangle \neq N \in \mathcal{N}$, then

$$
[a, b] \in N
$$

Let $1 \neq g=a^{\alpha} b^{\beta} c^{\gamma} d^{\delta} r^{\rho} s^{\zeta}[a, b]^{\eta} \in N$, where $0 \leq \alpha, \beta, \gamma, \delta, \rho, \zeta, \eta<p$. Since $r^{-1} g r=g[a, b]^{\zeta} \in N$, we obtain that $\zeta=0$, if $[a, b] \notin N$. Therefore, $g=a^{\alpha} b^{\beta} c^{\gamma} d^{\delta} r^{\rho}[a, b]^{\eta}$ and $b^{-1} g b=g[a, b]^{\alpha}$ now yields $\alpha=0$, if $[a, b] \notin N$. Proceeding similarly, we obtain that if $[a, b] \notin N$, then $g=1$, which contradicts the assumption and proves (3.1).

We next check that if $a \notin N$, then

$$
N=\langle[a, b]\rangle \text { or }\left\langle[a, b], r a^{i}\right\rangle \text {, where } 0 \leq i<p \text {. }
$$

In view of (3.1), we already have that either $N=\langle[a, b]\rangle$ or there exists an element $g=a^{\alpha} b^{\beta} c^{\gamma} d^{\delta} r^{\rho} s^{\zeta} \in N \backslash\langle[a, b]\rangle$, where $0 \leq \alpha, \beta, \gamma, \delta, \rho, \zeta<p$. Observe that $d^{-1} g d=g[a, b]^{\gamma} a^{-\zeta} \in N$ implies $\zeta=0$, if $a \notin N$. Continuing in this manner, repeated conjugacy by $s$ implies $\delta=\gamma=\beta=0$, if $a \notin N$ and $g=a^{\alpha} r^{\rho}, \rho \neq 0$. Thus, (3.2) follows. 
Next, assume $a \in N$, so that $\left\langle a, b^{-1} a b\right\rangle(\in \mathcal{N}) \leq N$, i.e., we look for normal subgroups $N$ of $G$ containing $\left\langle a, b^{-1} a b\right\rangle$, which are in one-one correspondence with the normal subgroups $\bar{N}$ of $\bar{G}:=G /\left\langle a, b^{-1} a b\right\rangle$. Proceeding as previously, we see that if $\overline{1} \neq \bar{g}=\bar{b}^{\beta} \bar{c}^{\gamma} \bar{d}^{\delta} \bar{r}^{\rho} \bar{s}^{\zeta} \in \bar{N}$, for some $0 \leq \beta, \gamma, \delta, \rho, \zeta<p$, then either $\bar{d} \in \bar{N}$ or $\bar{g}=\bar{d}^{\delta} \bar{r}^{\rho}, \rho \neq 0$, and in the later case,

$$
N=\left\langle a, b^{-1} a b\right\rangle \text { or }\left\langle a, b^{-1} a b, r d^{i}\right\rangle, \text { where } 0 \leq i<p .
$$

Now, if $\bar{d} \in \bar{N}$, then $\bar{N}$ is a normal subgroup of $\bar{G}$, which contains the normal subgroup $\langle\bar{d}\rangle$ of $\bar{G}$. Hence, $\bar{N}$ corresponds to a normal subgroup $\overline{\bar{N}}$ of $\overline{\bar{G}}:=\bar{G} /\langle\bar{d}\rangle$. Continuing this process, the set of normal subgroups of $G$, is obtained as

$$
\mathcal{N}=\left\{G, N_{0}, N_{1}, N_{2}, N_{3}, N_{4}, N_{5}, N_{6}, G_{i}^{(1)}, G_{i}^{(2)}, G_{i}^{(3)}, G_{i}^{(4)}, G_{i}^{(5)}, H_{i j}, K_{i j} \mid 0 \leq i, j<p\right\},
$$

where

$$
\begin{aligned}
& N_{0}:=\langle 1\rangle ; N_{1}:=\langle a, b, c, d, r\rangle ; N_{2}:=\langle a, b, c, d\rangle ; N_{3}:=\langle a, c, d\rangle ; \\
& N_{4}:=\left\langle a, b^{-1} a b, d\right\rangle ; N_{5}:=\left\langle a, b^{-1} a b\right\rangle ; N_{6}:=\langle[a, b]\rangle ; \\
& G_{i}^{(1)}:=\left\langle a, b, c, d, s r^{i}\right\rangle ; G_{i}^{(2)}:=\left\langle a, c, d, r b^{i}\right\rangle ; G_{i}^{(3)}:=\left\langle a, b^{-1} a b, d, r c^{i}\right\rangle ; \\
& G_{i}^{(4)}:=\left\langle a, b^{-1} a b, r d^{i}\right\rangle ; G_{i}^{(5)}:=\left\langle[a, b], r a^{i}\right\rangle ; \\
& H_{i j}:=\left\langle a, c, d, r b^{i}, s b^{j}\right\rangle ; \text { and } K_{i j}:=\left\langle a, c, d, s r^{i} b^{j}\right\rangle, \text { for } 0 \leq i, j<p .
\end{aligned}
$$

Note that if $N=G, N_{1}, H_{i j}, G_{i}^{(1)}, N_{2}, K_{i j}, G_{i}^{(2)}$ or $N_{3}$, then $G / N$ is abelian and hence, by Eq. (2.1), we have

$$
\mathcal{S}_{N}= \begin{cases}\{(G, N)\}, & \text { if } N \in \mathcal{N}_{1}:=\left\{G, N_{1}, H_{i j}, G_{i}^{(1)}\right\}, \\ \emptyset, & \text { if } N \in \mathcal{N}_{2}:=\left\{N_{2}, K_{i j}, G_{i}^{(2)}, N_{3}\right\} .\end{cases}
$$

Further, if $N \neq N_{0}$ is such that $G / N$ is not abelian, then $N_{1} / N$ is abelian. Consequently,

$$
A_{N}=N_{1}, \text { if } N \in \mathcal{N}_{3}:=\left\{G_{i}^{(3)}, N_{4}, G_{i}^{(4)}, N_{5}, G_{i}^{(5)}, N_{6}\right\}
$$

Also, it is easy to observe that

$$
A_{N}=G_{0}^{(3)}, \text { if } N \in \mathcal{N}_{4}:=\left\{N_{0}\right\}
$$

serves the purpose.

Since $\mathcal{N}=\mathcal{N}_{1} \cup \mathcal{N}_{2} \cup \mathcal{N}_{3} \cup \mathcal{N}_{4}$, in view of (3.5)-(3.7), we need to find $\mathcal{T}_{N}$, when $N \in \mathcal{N}_{3} \cup \mathcal{N}_{4}$. We first take up the case when $N \in \mathcal{N}_{4}$, i.e., $N=N_{0}=\langle 1\rangle$. In this case, since $A_{N_{0}}=G_{0}^{(3)}$ is an abelian p-group, the set $\mathcal{C}_{0}$ of subgroups of $A_{N_{0}}$, 
which give cyclic quotient can be computed using the algorithm given in Section 5 of [10] and is as follows:

$$
\mathcal{C}_{0}=\left\{H^{(0)}, H^{(1)}, H_{\beta}, H_{\alpha \beta}, H_{\alpha \beta \delta} \mid 0 \leq \alpha, \beta, \delta<p\right\},
$$

where $H^{(0)}:=\left\langle a, d, r, b^{-1} a b\right\rangle, \quad H^{(1)}:=\langle a, d, r\rangle, \quad H_{\beta}:=\left\langle d, r, b^{-1} a b a^{\beta}\right\rangle$, $H_{\alpha \beta}:=\left\langle a d^{\alpha}, r, b^{-1} a b d^{\beta}\right\rangle, H_{\alpha \beta \delta}:=\left\langle a r^{\alpha}, b^{-1} a b r^{\beta}, d r^{\delta}\right\rangle$, for $0 \leq \alpha, \beta, \delta<p$. In view of (3.1), we note that for $H \in \mathcal{C}_{0}, \operatorname{core}_{G}(H)=N_{0}=\langle 1\rangle$, if, and only if, $[a, b] \notin H$. This yields,

$$
\mathcal{D}_{N_{0}}=\left\{H^{(1)}\right\} \cup\left\{H_{\beta} \mid \beta \neq-1\right\} \cup\left\{H_{\alpha \beta}, H_{\alpha \beta \delta} \mid \alpha \neq \beta, 0 \leq \alpha, \beta, \delta<p\right\} .
$$

It turns out that all subgroups of $\mathcal{D}_{N_{0}}$ are $G$-conjugates and hence

$$
\mathcal{T}_{N_{0}}=\left\{H^{(1)}\right\}
$$

For ease of the reader, we list the conjugating element $g \in G$ such that $H^{g}=H^{(1)}$, for every $H \in \mathcal{D}_{N_{0}}$.

- $H_{\beta}^{x}=H^{(1)}$, with $x=b^{\nu}$, where $\nu(\beta+1) \equiv-1(\bmod p), \beta \neq-1$.

- $H_{\alpha \beta}^{y}=H^{(1)}$, with $y=\left(b^{\alpha} c\right)^{\lambda}$, where $\lambda(\beta-\alpha) \equiv 1(\bmod p), \alpha \neq \beta$.

- $H_{\alpha \beta \delta}^{z}=H^{(1)}$, with $z=\left(b^{\alpha} c^{-\delta}\right)^{\lambda} s^{\lambda}$, where $\lambda(\beta-\alpha) \equiv 1(\bmod p), \alpha \neq \beta$.

Finally, we proceed to find $\mathcal{S}_{N}$, when $N \in \mathcal{N}_{3}$. For this, we find the set $\mathcal{C}_{1}$ of subgroups of $A:=N_{1}=\langle a, b, c, d, r\rangle$ ( see (3.6) ) which give cyclic quotient, so that

$$
\mathcal{D}_{N}:=\left\{D \in \mathcal{C}_{1} \mid \operatorname{core}_{G}(D)=N\right\}
$$

Let $D \in \mathcal{C}_{1}$. Then, $A / D$ being cyclic ensures $[a, b] \in D$. Moreover, any element $\bar{g}=\bar{a}^{\alpha} \bar{b}^{\beta} \bar{c}^{\gamma} \bar{d}^{\delta} \bar{r}^{\rho} \in \bar{A}:=A / D$, where $0 \leq \alpha, \beta, \gamma, \delta, \rho<p$, is such that $\bar{g}^{p}=\overline{1}$, so that $\bar{A}=\langle\overline{1}\rangle$ or a cyclic group of order $p$. If $a \notin D$, then $\bar{a} \in \bar{A}$ yields $\bar{A}=\langle\bar{a}\rangle$. Also, $\bar{b} \in \bar{A}$ implies $b a^{\beta} \in D$, for some $0 \leq \beta<p$. Similarly, $c a^{\gamma}, r a^{\rho}, d a^{\delta} \in D$, for some $0 \leq \gamma, \rho, \delta<p$. This, along with (3.1) and order considerations yield $D=D_{\rho \delta \gamma \beta}:=\left\langle[a, b], r a^{\rho}, d a^{\delta}, c a^{\gamma}, b a^{\beta}\right\rangle$, for some $0 \leq \rho, \delta, \gamma, \beta<p$. Assuming next, $a \in D$ and $b \notin D$ gives $D=D_{\rho \delta \gamma}:=\left\langle a,[a, b], r b^{\rho}, d b^{\delta}, c b^{\gamma}\right\rangle, 0 \leq \rho, \delta, \gamma<p$. Iterating in the above manner, we obtain

$$
\mathcal{C}_{1}:=\left\{D^{(0)}, D^{(1)}, D_{\rho}, D_{\rho \delta}, D_{\rho \delta \gamma}, D_{\rho \delta \gamma \beta} \mid 0 \leq \rho, \delta, \gamma, \beta<p\right\}
$$

where $D^{(0)}:=A, D^{(1)}:=\langle a, b, c, d\rangle, D_{\rho}:=\left\langle a, b, c, r d^{\rho}\right\rangle, D_{\rho \delta}:=\left\langle a, b, d c^{\delta}, r c^{\rho}\right\rangle$, and $D_{\rho \delta \gamma}, D_{\rho \delta \gamma \beta}$ are as defined above, $0 \leq \rho, \delta, \gamma, \beta<p$. Since core ${ }_{G}(D)$ is the largest element of $\mathcal{N}$, contained in $D$, in view of (3.4), (3.10) \& (3.11), we obtain: 
- $\mathcal{D}_{N}=\emptyset$, if $N=N_{4}, N_{5}$ or $N_{6}$.

- $\mathcal{D}_{N}=\left\{D_{i 0}\right\} \cup\left\{D_{\rho 0 \gamma} \mid \rho \equiv-\gamma i(\bmod p), 0<\gamma<p\right\}$, if $N=G_{i}^{(3)}, 0 \leq i<p$.

- $\mathcal{D}_{N}=\left\{D_{i}\right\} \cup\left\{D_{\rho \delta}, D_{\rho \delta \gamma} \mid \rho \equiv-\delta i(\bmod p), 0<\delta<p, 0 \leq \gamma<p\right\}$, if $N=G_{i}^{(4)}, 0 \leq i<p$.

- $\mathcal{D}_{N}=\left\{D_{i \delta \gamma \beta} \mid 0 \leq \delta, \gamma, \beta<p\right\}$, if $N=G_{i}^{(5)}, 0 \leq i<p$.

We now omit the details for the remaining part and enlist the set $\mathcal{T}_{N}$, when $N=G_{i}^{(3)}, G_{i}^{(4)}, G_{i}^{(5)}, 0 \leq i<p$.

- $\mathcal{T}_{N}=\left\{D_{i 0}\right\}$, if $N=G_{i}^{(3)}, 0 \leq i<p$.

- $\mathcal{T}_{N}=\left\{D_{i}\right\} \cup\left\{D_{i \delta 0} \mid 0<\delta<p\right\}$, if $N=G_{i}^{(4)}, \quad 0 \leq i<p$.

- $\mathcal{T}_{N}=\left\{D_{i 0 \gamma \beta}, 0 \leq \gamma, \beta<p\right\}$, if $N=G_{i}^{(5)}, 0 \leq i<p$.

In view of (2.2) and Eq. (2.6), the information gathered above yields the complete set of strong Shoda pairs of $G$, and their respective simple components, as tabulated below:

\begin{tabular}{|c|c|c|c|c|}
\hline Normal Subgroup, $N$ & $A_{N}$ & $\mathcal{S}_{N}$ & $\left|\mathcal{R}\left(A_{N} / D\right)\right|$ & $\bigoplus_{(A, D) \in \mathcal{S}_{N}} \mathbb{F}_{q} G e_{\mathcal{C}}\left(G, A_{N}, D\right)$ \\
\hline$G$ & $G$ & $\{(G, G)\}$ & 1 & $\mathbb{F}_{q}$ \\
\hline$N_{1}=\langle a, b, c, d, r\rangle$ & $G$ & $\left\{\left(G, N_{1}\right)\right\}$ & $\frac{p-1}{f}$ & $\mathbb{F}_{q^{f}}$ \\
\hline $\begin{aligned} H_{i, j} & =\left\langle a, c, d, r b^{i}, s b^{j}\right\rangle \\
& 0 \leq i, j<p\end{aligned}$ & $G$ & $\left\{\left(G, H_{i j}\right)\right\}$ & $\frac{p-1}{f}$ & $\begin{array}{c}\mathbb{F}_{q^{f}} \\
0 \leq i, j<p\end{array}$ \\
\hline $\begin{aligned} G_{i}^{(1)}= & \left\langle a, b, c, d, s r^{i}\right\rangle \\
& 0 \leq i<p\end{aligned}$ & $G$ & $\left\{\left(G, G_{i}^{(1)}\right)\right\}$ & $\frac{p-1}{f}$ & $\begin{array}{c}\mathbb{F}_{q^{f}} \\
0 \leq i<p\end{array}$ \\
\hline$N_{2}=\langle a, c, d, b\rangle$ & $G$ & $\emptyset$ & - & - \\
\hline $\begin{array}{c}K_{i, j}=\left\langle a, c, d, s r^{i} b^{j}\right\rangle \\
0 \leq i, j<p\end{array}$ & $G$ & $\emptyset$ & - & - \\
\hline $\begin{aligned} G_{i}^{(2)}= & \left\langle a, c, d, r b^{i}\right\rangle \\
& 0 \leq i<p\end{aligned}$ & $G$ & $\emptyset$ & - & - \\
\hline$N_{3}=\langle a, c, d\rangle$ & $G$ & $\emptyset$ & - & - \\
\hline $\begin{aligned} G_{i}^{(3)}= & \left\langle a, d, b^{-1} a b, r c^{i}\right\rangle, \\
& 0 \leq i<p\end{aligned}$ & $N_{1}$ & $\left\{\left(N_{1},\left\langle a, b, d, r c^{i}\right\rangle\right)\right\}$ & $\frac{p-1}{f}$ & $\begin{array}{l}M_{p}\left(\mathbb{F}_{q^{f}}\right) \\
0 \leq i<p\end{array}$ \\
\hline$N_{4}=\left\langle a, b^{-1} a b, d\right\rangle$ & $N_{1}$ & $\emptyset$ & - & - \\
\hline $\begin{aligned} G_{i}^{(4)}= & \left\langle a, b^{-1} a b, r d^{i}\right\rangle \\
& 0 \leq i<p\end{aligned}$ & $N_{1}$ & $\begin{array}{l}\left\{\left(N_{1},\left\langle a, b, c, r d^{i}\right\rangle\right)\right\} \cup \\
\left\{\left(N_{1},\left\langle a, b^{-1} a b, c, d b^{\delta}, r d^{i}\right\rangle\right) \mid 1 \leq \delta<p\right\}\end{array}$ & $\frac{p-1}{f}$ & $\begin{array}{c}M_{p}\left(\mathbb{F}_{q_{f}}\right)^{(p)} \\
0 \leq i<p\end{array}$ \\
\hline$N_{5}=\left\langle a, b^{-1} a b\right\rangle$ & $N_{1}$ & $\emptyset$ & - & - \\
\hline $\begin{aligned} G_{i}^{(5)} & =\left\langle[a, b], r a^{i}\right\rangle \\
& 0 \leq i<p\end{aligned}$ & $N_{1}$ & $\left\{\left(N_{1},\left\langle[a, b], d, c a^{\gamma}, b a^{\beta}, r a^{i}\right\rangle\right) \mid 0 \leq \beta, \gamma<p\right\}$ & $\frac{p-1}{f}$ & $\begin{array}{c}M_{p}\left(\mathbb{F}_{q^{f}}\right)^{\left(p^{2}\right)} \\
0 \leq i<p\end{array}$ \\
\hline$N_{6}=\langle[a, b]\rangle$ & $N_{1}$ & $\emptyset$ & - & - \\
\hline$N_{0}=\langle 1\rangle$ & $G_{0}^{(3)}$ & $\left\{\left(\left\langle a, d, r, b^{-1} a b\right\rangle,\langle a, d, r\rangle\right)\right\}$ & $\frac{p-1}{f}$ & $M_{p^{3}}\left(\mathbb{F}_{q^{f}}\right)$ \\
\hline
\end{tabular}

Hence, the desired result is obtained. 
Remarks. Let $G$ be as in statement of Theorem 3. Then, by the irredundant set of strong Shoda pairs of $G$, obtained in Theorem 3 and ([5], Corollary 1), it follows that

$$
\mathbb{Q} G \cong \mathbb{Q} \bigoplus \mathbb{Q}\left(\zeta_{p}\right)^{\left(1+p+p^{2}\right)} \bigoplus M_{p}\left(\mathbb{Q}\left(\zeta_{p}\right)\right)^{\left(p\left(1+p+p^{2}\right)\right)} \bigoplus M_{p^{3}}\left(\mathbb{Q}\left(\zeta_{p}\right)\right)
$$

A complete irredundant set of strong Shoda pairs of $G$ for the case when $p=5$ has been computed in [5], using GAP [14], to find the Wedderburn Decomposition of the rational group algebra $\mathbb{Q} G$. However, it may be pointed out that GAP package Wedderga [12] fails to compute the Wedderburn decomposition of $\mathbb{Q} G$ or $\mathbb{F}_{q} G$, for any $p \geq 5$. In fact, though theoretically, Wedderga [12] could handle the calculation of the Wedderburn decomposition of group algebras of groups of arbitrary size but in practice, if the order of the group is greater than 5000 then the program may crash. Wedderga features the function StrongShodaPairs (G); that determines a complete irredundant set of strong Shoda pairs of $G$ and the function PrimitiveCentralIdempotentsByStrongSP (FG); that computes the set of primitive central idempotents of semisimple group algebra $\mathbb{F} G$. For the case when $\mathbb{F}=\mathbb{Q}$, these functions are based on the search algorithms provided by Olivieri and del Río [20]. Based on the work in [5], alternative and more efficient algorithms have been given in [7]. Analogously, in view of Theorem 1, improved algorithms can be written and implemented in GAP, for the case when $\mathbb{F}$ is a finite field.

\subsection{Groups of order $p^{n}, p$ prime, $n<5$}

The groups of order $p^{n}, p$ prime, $n<5$ are metabelian and hence normally monomial. Therefore, by applying Theorem 1, we now give the explicit structure of $\mathbb{F}_{q} G$, and its automorphism group when $G$ is a group of order $p^{n}$, $p$ prime, $n<5$. The Wedderburn decomposition of $\mathbb{F}_{q} G$, when $G$ is abelian is well known [11] and the automorphism group in this case can be computed as in ([1], Theorem 4). We therefore restrict to the case when $G$ is non-abelian.

\subsubsection{Groups of order $p^{3}$}

$\underline{p=2}$

If $p=2$, then $G$ is either $Q_{8}$, the quaternion group of order 8 or $D_{8}$, the dihedral group of order 8 . In this case, the set of primitive central idempotents and the Wedderburn decomposition of $\mathbb{F}_{q} G$ can be read from ([2], Examples 4.3 \& 4.4). 
In fact,

$$
\mathbb{F}_{q} Q_{8} \cong \mathbb{F}_{q} D_{8} \cong \mathbb{F}_{q}^{(4)} \bigoplus M_{2}\left(\mathbb{F}_{q}\right)
$$

and it follows from ([1], Theorem 5) that

$$
\operatorname{Aut}\left(\mathbb{F}_{q} Q_{8}\right) \cong \operatorname{Aut}\left(\mathbb{F}_{q} D_{8}\right) \cong S_{4} \bigoplus S L_{2}\left(\mathbb{F}_{q}\right)
$$

$\underline{p \neq 2}$

If $p \neq 2$, then $G$ is isomorphic to either $\left\langle a, b \mid a^{p^{2}}=b^{p}=1, b^{-1} a b=a^{1+p}\right\rangle$ or $\left\langle a, b, c \mid a^{p}=b^{p}=c^{p}=1, a b=b a c, a c=c a, b c=c b\right\rangle([15], \S 4.4)$. The strong Shoda pairs of each of these groups, found in ([5], Theorems $3 \& 4$ ) along with Theorem 1, Eq. (2.6) and ([1], Theorem 5), yield the following:

Theorem 4 Let $G$ be a non-abelian group of order $p^{3}$, where $p$ is an odd prime. Then,

$$
\mathbb{F}_{q} G \cong \mathbb{F}_{q} \bigoplus \mathbb{F}_{q^{f}}{ }^{((1+p) e)} \bigoplus M_{p}\left(\mathbb{F}_{q^{f}}\right)^{(e)}
$$

and

$$
\operatorname{Aut}\left(\mathbb{F}_{q} G\right) \cong\left\{\begin{array}{ll}
\left(\mathbb{Z}_{f}^{((1+p) e)} \rtimes S_{(1+p) e}\right) \bigoplus\left(\left(S L_{p}\left(\mathbb{F}_{q^{f}}\right) \rtimes \mathbb{Z}_{f}\right)^{(e)} \rtimes S_{e}\right), & f \neq 1 \\
S_{(2+p) e} \bigoplus\left(S L_{p}\left(\mathbb{F}_{q}\right)^{(e)} \rtimes S_{e}\right), & f=1
\end{array},\right.
$$

where $f=o_{p}(q)$ and $e=\frac{p-1}{f}$.

\subsubsection{Groups of order $p^{4}$}

$\underline{p=2}$

If $p=2$, then up to isomorphism, there are 9 non-isomorphic groups of order $2^{4}([13], \S 118)$ as listed below:

$$
\begin{aligned}
& \mathcal{H}_{1}:=\left\langle a, b: a^{8}=b^{2}=1, b a=a^{5} b\right\rangle ; \\
& \mathcal{H}_{2}:=\left\langle a, b, c: a^{4}=b^{2}=c^{2}=1, c b=a^{2} b c, a b=b a, a c=c a\right\rangle ; \\
& \mathcal{H}_{3}:=\left\langle a, b: a^{4}=b^{4}=1, b a=a^{3} b\right\rangle ; \\
& \mathcal{H}_{4}:=\left\langle a, b, c: a^{4}=b^{2}=c^{2}=1, c a=a^{3} c, b a=a b, c b=b c\right\rangle ; \\
& \mathcal{H}_{5}:=\left\langle a, b, c: a^{4}=b^{2}=c^{2}=1, c a=a b c, b a=a b, c b=b c\right\rangle ; \\
& \mathcal{H}_{6}:=\left\langle a, b, c: a^{4}=b^{4}=c^{2}=1, b a=a^{3} b, c a=a c, c b=b c, a^{2}=b^{2}\right\rangle ;
\end{aligned}
$$




$$
\begin{aligned}
& \mathcal{H}_{7}:=\left\langle a, b: a^{8}=b^{2}=1, b a=a^{7} b\right\rangle ; \\
& \mathcal{H}_{8}:=\left\langle a, b: a^{8}=b^{2}=1, b a=a^{3} b\right\rangle ; \\
& \mathcal{H}_{9}:=\left\langle a, b: a^{8}=b^{4}=1, b a=a^{7} b, a^{4}=b^{2}\right\rangle .
\end{aligned}
$$

Theorem 5 The Wedderburn decomposition of $\mathbb{F}_{q} \mathcal{H}_{i}, 1 \leq i \leq 9$, is as follows:

(i) $\mathbb{F}_{q} \mathcal{H}_{1} \cong\left\{\begin{array}{ll}\mathbb{F}_{q}{ }^{(8)} \bigoplus M_{2}\left(\mathbb{F}_{q}\right)^{(2)} & q \equiv 1,5(\bmod 8) \\ \mathbb{F}_{q}{ }^{(4)} \bigoplus \mathbb{F}_{q^{2}}{ }^{(2)} \bigoplus M_{2}\left(\mathbb{F}_{q^{2}}\right) & q \equiv 3,7(\bmod 8)\end{array} ;\right.$

(ii) $\mathbb{F}_{q} \mathcal{H}_{2} \cong\left\{\begin{array}{ll}\mathbb{F}_{q}{ }^{(8)} \bigoplus M_{2}\left(\mathbb{F}_{q}\right)^{(2)} & q \equiv 1(\bmod 4) \\ \mathbb{F}_{q}{ }^{(8)} \bigoplus M_{2}\left(\mathbb{F}_{q^{2}}\right) & q \equiv 3(\bmod 4)\end{array} ;\right.$

(iii) $\mathbb{F}_{q} \mathcal{H}_{3} \cong\left\{\begin{array}{ll}\mathbb{F}_{q}{ }^{(8)} \bigoplus M_{2}\left(\mathbb{F}_{q}\right)^{(2)} & q \equiv 1(\bmod 4) \\ \mathbb{F}_{q}{ }^{(4)} \bigoplus \mathbb{F}_{q^{2}}{ }^{(2)} \bigoplus M_{2}\left(\mathbb{F}_{q}\right)^{(2)} & q \equiv 3(\bmod 4)\end{array} ;\right.$

(iv) $\mathbb{F}_{q} \mathcal{H}_{4} \cong \mathbb{F}_{q}^{(8)} \bigoplus M_{2}\left(\mathbb{F}_{q}\right)^{(2)}$;

$(\mathbf{v}) \mathbb{F}_{q} \mathcal{H}_{5} \cong\left\{\begin{array}{ll}\mathbb{F}_{q}^{(8)} \bigoplus M_{2}\left(\mathbb{F}_{q}\right)^{(2)} & q \equiv 1(\bmod 4) \\ \mathbb{F}_{q}^{(4)} \bigoplus \mathbb{F}_{q^{2}}{ }^{(2)} \bigoplus M_{2}\left(\mathbb{F}_{q}\right)^{(2)} & q \equiv 3(\bmod 4)\end{array} ;\right.$

(vi) $\mathbb{F}_{q} \mathcal{H}_{6} \cong \mathbb{F}_{q}^{\left({ }^{(8)}\right.} \bigoplus M_{2}\left(\mathbb{F}_{q}\right)^{(2)}$;

(vii) $\mathbb{F}_{q} \mathcal{H}_{7} \cong\left\{\begin{array}{ll}\mathbb{F}_{q}{ }^{(4)} \bigoplus M_{2}\left(\mathbb{F}_{q}\right)^{(3)} & q \equiv 1,7(\bmod 8) \\ \mathbb{F}_{q}{ }^{(4)} \bigoplus M_{2}\left(\mathbb{F}_{q^{2}}\right) \oplus M_{2}\left(\mathbb{F}_{q}\right) & q \equiv 3,5(\bmod 8)\end{array} ;\right.$

(viii) $\mathbb{F}_{q} \mathcal{H}_{8} \cong \begin{cases}\mathbb{F}_{q}^{(4)} \oplus M_{2}\left(\mathbb{F}_{q}\right)^{(3)} & q \equiv 1,3(\bmod 8) \\ \mathbb{F}_{q}^{(4)} \oplus M_{2}\left(\mathbb{F}_{q^{2}}\right) \oplus M_{2}\left(\mathbb{F}_{q}\right) & q \equiv 5,7(\bmod 8)\end{cases}$

(ix) $\mathbb{F}_{q} \mathcal{H}_{9} \cong \begin{cases}\mathbb{F}_{q}^{(4)} \bigoplus M_{2}\left(\mathbb{F}_{q}\right)^{(3)} & q \equiv 1,7(\bmod 8) \\ \mathbb{F}_{q}{ }^{(4)} \bigoplus M_{2}\left(\mathbb{F}_{q^{2}}\right) \bigoplus M_{2}\left(\mathbb{F}_{q}\right) & q \equiv 3,5(\bmod 8)\end{cases}$

Proof. (i) Define $N_{0}:=\langle 1\rangle, N_{1}:=\left\langle a^{4}\right\rangle, N_{2}:=\left\langle a^{2}\right\rangle, N_{3}:=\langle a\rangle, H_{i}:=\left\langle a^{4}, a^{2 i} b\right\rangle$, $K_{j}:=\left\langle a^{2}, a^{j} b\right\rangle$ where $0 \leq i, j \leq 1$. Observe that these subgroups are normal in $\mathcal{H}_{1}$. Using Eq. (2.1), we have $\mathcal{S}_{N_{1}}=\mathcal{S}_{N_{2}}=\phi, \mathcal{S}_{N_{3}}=\left\{\left(\mathcal{H}_{1}, N_{3}\right)\right\}, \mathcal{S}_{H_{i}}=\left\{\left(\mathcal{H}_{1}, H_{i}\right)\right\}$, $\mathcal{S}_{K_{j}}=\left\{\left(\mathcal{H}_{1}, K_{j}\right)\right\}, 0 \leq i, j \leq 1$. In order to find $\mathcal{S}_{N_{0}}$, we see that $\langle a\rangle$ is a maximal abelian subgroup of $\mathcal{H}_{1}$. Further, the only subgroup $D$ of $\langle a\rangle$ satisfying $\operatorname{core}_{G}(D)=\langle 1\rangle$ is $D=\langle 1\rangle$. This gives $\mathcal{S}_{N_{0}}=\{(\langle a\rangle,\langle 1\rangle)\}$. Define

$$
\mathcal{N}_{1}=\left\{\langle 1\rangle,\left\langle a^{4}\right\rangle,\left\langle a^{2}\right\rangle,\langle a\rangle,\langle a, b\rangle\right\} \cup\left\{\left\langle a^{4}, a^{2 i} b\right\rangle,\left\langle a^{2}, a^{j} b\right\rangle \mid 0 \leq i, j \leq 1\right\} .
$$

It follows from Eqs. (2.3)-(2.4) that $\bigoplus_{N \in \mathcal{N}_{1}} \bigoplus_{\left(A_{N}, D\right) \in \mathcal{S}_{N}} \bigoplus_{C \in \mathcal{R}\left(A_{N} / D\right)} \mathbb{F}_{q} G e_{C}\left(G, A_{N}, D\right)$, is a direct summand of $\mathbb{F}_{q} G$ and has same $\mathbb{F}_{q}$-dimension as $\mathbb{F}_{q} G$. This yields that 
if $\mathcal{N}$ is the set of all normal subgroups of $\mathcal{H}_{1}$, then $\mathcal{S}_{N}=\phi$, if $N \notin \mathcal{N}_{1}$, i.e., $\mathcal{S}\left(\mathcal{H}_{1}\right)=\bigcup_{N \in \mathcal{N}_{1}} \mathcal{S}_{N}$ and

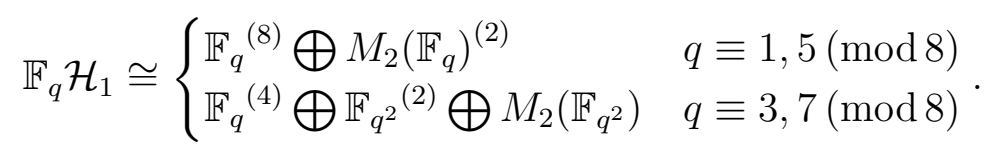

(ii)-(ix) For $2 \leq i \leq 9$, consider the following set $\mathcal{N}_{i}$ of normal subgroups of $\mathcal{H}_{i}$ :

$$
\begin{aligned}
\mathcal{N}_{2}= & \left\{\langle 1\rangle,\left\langle a^{2}\right\rangle,\left\langle a^{2}, b\right\rangle,\langle a, b\rangle,\langle a, b, c\rangle\right\} \cup \\
& \left\{\left\langle a^{2}, b^{i} c\right\rangle,\left\langle a, b^{i} c\right\rangle,\left\langle a b^{i} c^{j}\right\rangle,\left\langle a^{2}, a^{i} b, a^{j} c\right\rangle \mid 0 \leq i, j \leq 1\right\} ; \\
\mathcal{N}_{3}= & \left\{\langle 1\rangle,\left\langle a^{2}\right\rangle,\langle a\rangle,\left\langle a, b^{2}\right\rangle,\langle a, b\rangle\right\} \cup \\
& \left\{\left\langle a^{2 i} b^{2}\right\rangle,\left\langle a^{2}, a^{i} b\right\rangle,\left\langle a^{2}, a^{i} b^{2}\right\rangle \mid 0 \leq i \leq 1\right\} ; \\
\mathcal{N}_{4}= & \left\{\langle 1\rangle,\left\langle a^{2}\right\rangle,\left\langle a^{2}, b\right\rangle,\langle a, b\rangle,\langle a, b, c\rangle\right\} \cup \\
& \left\{\left\langle a^{2 i} b\right\rangle,\left\langle a^{2}, b^{i} c\right\rangle,\left\langle a, b^{i} c\right\rangle,\left\langle a^{2}, a b^{i} c^{j}\right\rangle,\left\langle a^{2}, a^{i} b, a^{j} c\right\rangle|0 \leq i, j \leq 1\rangle\right\} ; \\
\mathcal{N}_{5}= & \left\{\langle 1\rangle,\langle b\rangle,\left\langle a^{2}\right\rangle,\left\langle a^{2} b\right\rangle,\left\langle a^{2}, b\right\rangle,\langle a, b\rangle,\langle b, a c\rangle,\left\langle a^{2}, b, c\right\rangle,\langle a, b, c\rangle\right\} \cup \\
& \left\{\left\langle b, a^{2 i} c\right\rangle|0 \leq i \leq 1\rangle\right\} ; \\
\mathcal{N}_{6}= & \left\{\langle 1\rangle,\left\langle a^{2}\right\rangle,\left\langle a^{2}, c\right\rangle,\langle a, b\rangle,\langle a, b, c\rangle\right\} \cup \\
& \left\{\left\langle a^{i} c\right\rangle,\left\langle b c^{i}\right\rangle,\left\langle a b^{i} c^{j}\right\rangle,\left\langle a, b^{i} c\right\rangle,\left\langle a^{2}, a^{i} b, a^{j} c\right\rangle|0 \leq i, j \leq 1\rangle\right\} ; \\
\mathcal{N}_{7}= & \left\{\langle 1\rangle,\left\langle a^{4}\right\rangle,\left\langle a^{2}\right\rangle,\langle a\rangle,\langle a, b\rangle\right\} \cup\left\{\left\langle a^{2}, a^{i} b\right\rangle \mid 0 \leq i \leq 1\right\} ; \\
\mathcal{N}_{8}= & \left\{\langle 1\rangle,\left\langle a^{4}\right\rangle,\left\langle a^{2}\right\rangle,\langle a\rangle,\langle a, b\rangle\right\} \cup\left\{\left\langle a^{2}, a^{i} b\right\rangle \mid 0 \leq i \leq 1\right\} ; \\
\mathcal{N}_{9}= & \left\{\langle 1\rangle,\left\langle a^{4}\right\rangle,\left\langle a^{2}\right\rangle,\langle a\rangle,\langle a, b\rangle\right\} \cup\left\{\left\langle a^{2}, a^{i} b\right\rangle \mid 0 \leq i \leq 1\right\} .
\end{aligned}
$$

Proceeding as in (i), we get the following complete and irredundant set of strong Shoda pairs of $\mathcal{H}_{i}, 2 \leq i \leq 9$, which yield the desired result.

$$
\begin{aligned}
(\text { ii }) \mathcal{S}\left(\mathcal{H}_{2}\right)= & \left\{\left(\mathcal{H}_{2}, \mathcal{H}_{2}\right), \quad(\langle a, b\rangle,\langle b\rangle), \quad\left(\mathcal{H}_{2},\langle a, b\rangle\right)\right\} \cup \\
& \left\{\left(\mathcal{H}_{2},\left\langle a, b^{i} c\right\rangle\right), \quad\left(\mathcal{H}_{2},\left\langle a^{2}, a^{i} b, a^{j} c\right\rangle\right) \mid 0 \leq i, j \leq 1\right\} ; \\
(\text { iii }) \mathcal{S}\left(\mathcal{H}_{3}\right)= & \left\{\left(\mathcal{H}_{3}, \mathcal{H}_{3}\right), \quad\left(\mathcal{H}_{3},\left\langle a, b^{2}\right\rangle\right), \quad\left(\mathcal{H}_{3},\langle a\rangle\right), \quad\left(\mathcal{H}_{3},\left\langle a^{2}, a b^{2}\right\rangle\right)\right\} \cup \\
& \left\{\left(\left\langle a, b^{2}\right\rangle,\left\langle a^{2 i} b^{2}\right\rangle\right), \quad\left(\mathcal{H}_{3},\left\langle a^{2}, a^{i} b\right\rangle\right) \mid 0 \leq i \leq 1\right\} ; \\
(\text { iv }) \mathcal{S}\left(\mathcal{H}_{4}\right)= & \left\{\left(\mathcal{H}_{4}, \mathcal{H}_{4}\right), \quad\left(\mathcal{H}_{4},\langle a, b\rangle\right)\right\} \cup \\
& \left\{\left(\langle a, b\rangle,\left\langle a^{2 i} b\right\rangle\right), \quad\left(\mathcal{H}_{4},\left\langle a, b^{i} c\right\rangle\right), \quad\left(\mathcal{H}_{4},\left\langle a^{2}, a^{i} b, a^{j} c\right\rangle\right) \mid 0 \leq i, j \leq 1\right\} ; \\
(v) \mathcal{S}\left(\mathcal{H}_{5}\right)= & \left\{\left(\mathcal{H}_{5}, \mathcal{H}_{5}\right), \quad\left(\mathcal{H}_{5},\langle a, b\rangle\right), \quad\left(\mathcal{H}_{5},\left\langle a^{2}, b, c\right\rangle\right), \quad\left(\mathcal{H}_{5},\langle b, a c\rangle\right)\right\} \cup \\
& \left\{(\langle a, b\rangle,\langle a\rangle), \quad\left(\langle a, b\rangle,\left\langle a^{2} b\right\rangle\right)\right\} \cup\left\{\left(\mathcal{H}_{5},\left\langle b, a^{2 i} c\right\rangle\right) \mid 0 \leq i \leq 1\right\} ; \\
\text { (vi) } \mathcal{S}\left(\mathcal{H}_{6}\right)= & \left\{\left(\mathcal{H}_{6}, \mathcal{H}_{6}\right), \quad\left(\mathcal{H}_{6},\langle a, b\rangle\right)\right\} \cup \\
& \left\{\left(\langle a, c\rangle,\left\langle a^{i} c\right\rangle\right), \quad\left(\mathcal{H}_{6},\left\langle a, b^{i} c\right\rangle\right), \quad\left(\mathcal{H}_{6},\left\langle a^{2}, a^{i} b, a^{j} c\right\rangle\right) \mid 0 \leq i, j \leq 1\right\} ; \\
\text { (vii) } \mathcal{S}\left(\mathcal{H}_{7}\right)= & \left\{\left(\mathcal{H}_{7}, \mathcal{H}_{7}\right), \quad\left(\mathcal{H}_{7},\langle a\rangle\right)\right\} \cup \\
& \left\{\left(\langle a\rangle,\left\langle a^{4 i}\right\rangle\right), \quad\left(\mathcal{H}_{7},\left\langle a^{2}, a^{i} b\right\rangle\right) \mid 0 \leq i \leq 1\right\} ; \\
\text { (viii) } \mathcal{S}\left(\mathcal{H}_{8}\right)= & \left\{\left(\mathcal{H}_{8}, \mathcal{H}_{8}\right), \quad\left(\mathcal{H}_{8},\langle a\rangle\right)\right\} \cup \\
& \left\{\left(\langle a\rangle,\left\langle a^{4 i}\right\rangle\right), \quad\left(\mathcal{H}_{8},\left\langle a^{2}, a^{i} b\right\rangle\right) \mid 0 \leq i \leq 1\right\} ;
\end{aligned}
$$


(ix) $\mathcal{S}\left(\mathcal{H}_{9}\right)=\left\{\left(\mathcal{H}_{9}, \mathcal{H}_{9}\right), \quad\left(\mathcal{H}_{9},\langle a\rangle\right)\right\} \cup$

$\left\{\left(\langle a\rangle,\left\langle a^{4 i}\right\rangle\right), \quad\left(\mathcal{H}_{9},\left\langle a^{2}, a^{i} b\right\rangle\right) \mid 0 \leq i \leq 1\right\}$.

Corollary 1 The automorphism group of $\mathbb{F}_{q} \mathcal{H}_{i}, 1 \leq i \leq 9$, is as follows:

$$
\begin{aligned}
& \operatorname{Aut}\left(\mathbb{F}_{q} \mathcal{H}_{1}\right) \cong\left\{\begin{array}{ll}
S_{8} \bigoplus\left(S L_{2}\left(\mathbb{F}_{q}\right)^{(2)} \rtimes S_{2}\right), & q \equiv 1,5(\bmod 8) \\
S_{4} \bigoplus\left(\mathbb{Z}_{2}^{(2)} \rtimes S_{2}\right) \bigoplus\left(S L_{2}\left(\mathbb{F}_{q^{2}}\right) \rtimes \mathbb{Z}_{2}\right), & q \equiv 3,7(\bmod 8)
\end{array} ;\right. \\
& \operatorname{Aut}\left(\mathbb{F}_{q} \mathcal{H}_{2}\right) \cong\left\{\begin{array}{ll}
S_{8} \bigoplus\left(S L_{2}\left(\mathbb{F}_{q}\right)^{(2)} \rtimes S_{2}\right), & q \equiv 1(\bmod 4) \\
S_{8} \bigoplus\left(S L_{2}\left(\mathbb{F}_{q^{2}}\right) \rtimes \mathbb{Z}_{2}\right), & q \equiv 3(\bmod 4)
\end{array} ;\right. \\
& \operatorname{Aut}\left(\mathbb{F}_{q} \mathcal{H}_{3}\right) \cong\left\{\begin{array}{ll}
S_{8} \bigoplus\left(S L_{2}\left(\mathbb{F}_{q}\right)^{(2)} \rtimes S_{2}\right), & q \equiv 1(\bmod 4) \\
S_{4} \bigoplus\left(\mathbb{Z}_{2}{ }^{(2)} \rtimes S_{2}\right) \bigoplus\left(S L_{2}\left(\mathbb{F}_{q}\right)^{(2)} \rtimes S_{2}\right), & q \equiv 3(\bmod 4)
\end{array} ;\right. \\
& \operatorname{Aut}\left(\mathbb{F}_{q} \mathcal{H}_{4}\right) \cong S_{8} \bigoplus\left(S L_{2}\left(\mathbb{F}_{q}\right)^{(2)} \rtimes S_{2}\right) ; \\
& \operatorname{Aut}\left(\mathbb{F}_{q} \mathcal{H}_{5}\right) \cong\left\{\begin{array}{ll}
S_{8} \bigoplus\left(S L_{2}\left(\mathbb{F}_{q}\right)^{(2)} \rtimes S_{2}\right), & q \equiv 1(\bmod 4) \\
S_{4} \bigoplus\left(\mathbb{Z}_{2}{ }^{(2)} \rtimes S_{2}\right) \bigoplus\left(S L_{2}\left(\mathbb{F}_{q}\right)^{(2)} \rtimes S_{2}\right), & q \equiv 3(\bmod 4)
\end{array} ;\right. \\
& \operatorname{Aut}\left(\mathbb{F}_{q} \mathcal{H}_{6}\right) \cong S_{8} \bigoplus\left(S L_{2}\left(\mathbb{F}_{q}\right)^{(2)} \rtimes S_{2}\right) ; \\
& \operatorname{Aut}\left(\mathbb{F}_{q} \mathcal{H}_{7}\right) \cong\left\{\begin{array}{ll}
S_{4} \bigoplus\left(S L_{2}\left(\mathbb{F}_{q}\right)^{(3)} \rtimes S_{3}\right), & q \equiv 1,7(\bmod 8) \\
S_{4} \bigoplus\left(S L_{2}\left(\mathbb{F}_{q^{2}}\right) \rtimes \mathbb{Z}_{2}\right) \bigoplus S L_{2}\left(\mathbb{F}_{q}\right), & q \equiv 3,5(\bmod 8)
\end{array} ;\right. \\
& \operatorname{Aut}\left(\mathbb{F}_{q} \mathcal{H}_{8}\right) \cong\left\{\begin{array}{ll}
S_{4} \bigoplus\left(S L_{2}\left(\mathbb{F}_{q}\right)^{(3)} \rtimes S_{3}\right), & q \equiv 1,3(\bmod 8) \\
S_{4} \bigoplus\left(S L_{2}\left(\mathbb{F}_{q^{2}}\right) \rtimes \mathbb{Z}_{2}\right) \bigoplus S L_{2}\left(\mathbb{F}_{q}\right), & q \equiv 5,7(\bmod 8)
\end{array} ;\right. \\
& \operatorname{Aut}\left(\mathbb{F}_{q} \mathcal{H}_{9}\right) \cong\left\{\begin{array}{ll}
S_{4} \bigoplus\left(S L_{2}\left(\mathbb{F}_{q}\right)^{(3)} \rtimes S_{3}\right), & q \equiv 1,7(\bmod 8) \\
S_{4} \bigoplus\left(S L_{2}\left(\mathbb{F}_{q^{2}}\right) \rtimes \mathbb{Z}_{2}\right) \bigoplus S L_{2}\left(\mathbb{F}_{q}\right), & q \equiv 3,5(\bmod 8)
\end{array} .\right.
\end{aligned}
$$

$\underline{p \neq 2}$

If $p$ is an odd prime, then, up to isomorphism, the following are non-abelian groups of order $p^{4}([13], \S 117)$ :

$$
\begin{aligned}
& \mathcal{G}_{1}:=\left\langle a, b: a^{p^{3}}=b^{p}=1, b a=a^{1+p^{2}} b\right\rangle ; \\
& \mathcal{G}_{2}:=\left\langle a, b, c: a^{p^{2}}=b^{p}=c^{p}=1, c b=a^{p} b c, a b=b a, a c=c a\right\rangle ; \\
& \mathcal{G}_{3}:=\left\langle a, b: a^{p^{2}}=b^{p^{2}}=1, b a=a^{1+p} b\right\rangle ; \\
& \mathcal{G}_{4}:=\left\langle a, b, c: a^{p^{2}}=b^{p}=c^{p}=1, c a=a^{1+p} c, b a=a b, c b=b c\right\rangle ; \\
& \mathcal{G}_{5}:=\left\langle a, b, c: a^{p^{2}}=b^{p}=c^{p}=1, c a=a b c, a b=b a, b c=c b\right\rangle ;
\end{aligned}
$$




$$
\begin{aligned}
& \mathcal{G}_{6}:=\left\langle a, b, c: a^{p^{2}}=b^{p}=c^{p}=1, b a=a^{1+p} b, c a=a b c, c b=b c\right\rangle ; \\
& \mathcal{G}_{7}:=\left\{\begin{array}{l}
\left\langle a, b, c: a^{p^{2}}=b^{p}=1, c^{p}=a^{p}, a b=b a^{1+p}, a c=c a b^{-1}, c b=b c\right\rangle, \text { if } p=3, \\
\left\langle a, b, c: a^{p^{2}}=b^{p}=c^{p}=1, b a=a^{1+p} b, c a=a^{1+p} b c, c b=a^{p} b c\right\rangle, \text { if } p>3 ;
\end{array}\right. \\
& \mathcal{G}_{8}:=\left\{\begin{array}{l}
\left\langle a, b, c: a^{p^{2}}=b^{p}=1, c^{p}=a^{-p}, a b=b a^{1+p}, a c=c a b^{-1}, c b=b c\right\rangle, \text { if } p=3, \\
\left\langle a, b, c: a^{p^{2}}=b^{p}=c^{p}=1, b a=a^{1+p} b, c a=a^{1+d p} b c, c b=a^{d p} b c\right\rangle, \text { if } p>3
\end{array}\right. \\
& d \neq \equiv, 1(\bmod p) ;
\end{aligned}
$$$$
\mathcal{G}_{9}:=\left\langle a, b, c, d: a^{p}=b^{p}=c^{p}=d^{p}=1, d c=a c d, b d=d b, a d=d a, b c=c b,\right.
$$$$
a c=c a, a b=b a\rangle
$$$$
\mathcal{G}_{10}:=\left\{\begin{array}{c}
\left\langle a, b, c: a^{p^{2}}=b^{p}=c^{p}=1, a b=b a, a c=c a b, b c=c a^{-p} b\right\rangle, \text { if } p=3, \\
\left\langle a, b, c, d: a^{p}=b^{p}=c^{p}=d^{p}=1, d c=b c d, d b=a b d, a d=d a,\right. \\
b c=c b, a c=c a, a b=b a\rangle, \text { if } p>3 .
\end{array}\right.
$$

For $1 \leq i \leq 10$, a complete irredundant set $\mathcal{S}\left(\mathcal{G}_{i}\right)$ of strong Shoda pairs of $\mathcal{G}_{i}$ has been computed in ([6], Theorem 3) which in view of Theorem 1, and Eq. (2.6]) yields the following:

Theorem 6 The Wedderburn decomposition of $\mathbb{F}_{q} \mathcal{G}_{i}, 1 \leq i \leq 10$, is as follows:

$$
\begin{aligned}
& \mathbb{F}_{q} \mathcal{G}_{1} \cong \mathbb{F}_{q} \bigoplus \mathbb{F}_{q^{f}}((1+p) e) \bigoplus \mathbb{F}_{q^{f p}}{ }^{(p e)} \bigoplus M_{p}\left(\mathbb{F}_{q^{f p}}\right)^{(e)} \\
& \mathbb{F}_{q} \mathcal{G}_{2} \cong \mathbb{F}_{q} \bigoplus \mathbb{F}_{q^{f}}\left(\left(1+p+p^{2}\right) e\right) \bigoplus M_{p}\left(\mathbb{F}_{q^{f p}}\right)^{(e)} ; \\
& \mathbb{F}_{q} \mathcal{G}_{3} \cong \mathbb{F}_{q} \bigoplus \mathbb{F}_{q^{f}}{ }^{((1+p) e)} \bigoplus \mathbb{F}_{q^{f p}}{ }^{(e p)} \bigoplus M_{p}\left(\mathbb{F}_{q^{f}}\right)^{(p e)} ; \\
& \mathbb{F}_{q} \mathcal{G}_{4} \cong \mathbb{F}_{q} \bigoplus \mathbb{F}_{q^{f}}{ }^{\left(\left(1+p+p^{2}\right) e\right)} \bigoplus M_{p}\left(\mathbb{F}_{q^{f}}\right)^{(p e)} ; \\
& \mathbb{F}_{q} \mathcal{G}_{5} \cong \mathbb{F}_{q} \bigoplus \mathbb{F}_{q^{f}}{ }^{((1+p) e)} \bigoplus \mathbb{F}_{q^{f p}}{ }^{(p e)} \bigoplus M_{p}\left(\mathbb{F}_{q^{f}}\right)^{(p e)} \\
& \mathbb{F}_{q} \mathcal{G}_{6} \cong \mathbb{F}_{q} \bigoplus \mathbb{F}_{q^{f}}^{((1+p) e)} \bigoplus M_{p}\left(\mathbb{F}_{q^{f}}\right)^{((1+p) e)} ; \\
& \mathbb{F}_{q} \mathcal{G}_{7} \cong \mathbb{F}_{q} \bigoplus \mathbb{F}_{q^{f}}((1+p) e) \bigoplus M_{p}\left(\mathbb{F}_{q^{f}}\right)^{(e)} \bigoplus M_{p}\left(\mathbb{F}_{q^{f p}}\right)^{(e)} \\
& \mathbb{F}_{q} \mathcal{G}_{8} \cong \mathbb{F}_{q} \bigoplus \mathbb{F}_{q^{f}}((1+p) e) \bigoplus M_{p}\left(\mathbb{F}_{q^{f}}\right)^{(e)} \bigoplus M_{p}\left(\mathbb{F}_{q^{f p}}\right)^{(e)} ; \\
& \mathbb{F}_{q} \mathcal{G}_{9} \cong \mathbb{F}_{q} \bigoplus \mathbb{F}_{q^{f}}{ }^{\left(\left(1+p+p^{2}\right) e\right)} \bigoplus M_{p}\left(\mathbb{F}_{q^{f}}\right)^{(p e)} ;
\end{aligned}
$$

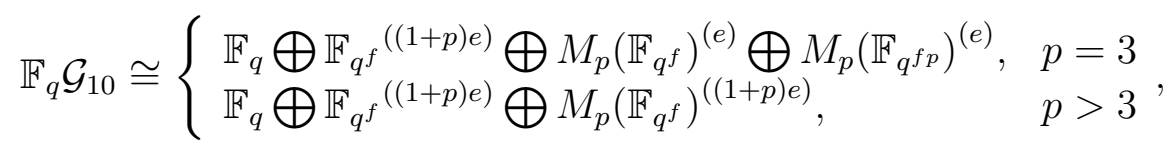

where $o_{p}(q)=f$ and $e=\frac{p-1}{f}$. 
The automorphism group of finite semisimple group algebra of groups of order $p^{4}, p$ an odd prime, can now be computed similarly.

\section{Acknowledgement}

The authors are grateful to I. B.S. Passi and G.K. Bakshi for their valuable comments and suggestions.

\section{References}

[1] G. K. Bakshi, S. Gupta, and I. B. S. Passi, Semisimple metacyclic group algebras, Proc. Indian Acad. Sci. Math. Sci. 121 (2011), no. 4, 379-396.

[2] _ The structure of semisimple metacyclic group algebras, J. Ramanujan Math. Soc. 28 (2013), no. 2, 141-158.

[3] _ , The algebraic structure of finite metabelian group algebras, Comm. Algebra 43 (2015), no. 6.

[4] G. K. Bakshi, R. S. Kulkarni, and I. B. S. Passi, The rational group algebra of a finite group, J. Algebra Appl. 12 (2013), no. 3.

[5] G. K. Bakshi and S. Maheshwary, The rational group algebra of a normally monomial group, J. Pure Appl. Algebra 218 (2014), no. 9, 1583-1593.

[6] _ On the index of a free abelian subgroup in the group of central units of an integral group ring, J. Algebra 434 (2015), 72-89.

[7] _ Extremely strong Shoda pairs with GAP, J. Symbolic Comput. 76 (2016), 97-106.

[8] G. K. Bakshi and M. Raka, Minimal cyclic codes of length $p^{n} q$, Finite Fields Appl. 9 (2003), no. 4, 432-448.

[9] G. K. Bakshi, M. Raka, and A. Sharma, Idempotent generators of irreducible cyclic codes, Number theory \& discrete geometry, Ramanujan Math. Soc. Lect. Notes Ser., vol. 6, Ramanujan Math. Soc., Mysore, 2008, pp. 13-18.

[10] B. G. Basmaji, Monomial representations and metabelian groups, Nagoya Math. J. 35 (1969), 99-107.

[11] O. Broche and Á. del Río, Wedderburn decomposition of finite group algebras, Finite Fields Appl. 13 (2007), no. 1, 71-79. 
[12] O. Broche Cristo, A. Herman, A. Konovalov, A. Olivieri, G. Olteanu, Á. del Río, and I. van Geldar, Wedderga - wedderburn decomposition of group algebras, Version 4.7.2; (2014), (http://www.cs.st-andrews.ac.uk/ ãlexk/wedderga).

[13] W. Burnside, Theory of groups of finite order, Dover Publications, Inc., New York, 1955, 2d ed.

[14] The GAP Group, GAP - Groups, Algorithms, and Programming, Version 4.7.8, 2015.

[15] M. Hall, Jr., The theory of groups, Chelsea Publishing Co., New York, 1976, Reprinting of the 1968 edition.

[16] G. A. How, Special classes of monomial groups. III, Chinese J. Math. 12 (1984), no. 3, 199-211.

[17] N. Makhijani, R. K. Sharma, and J. B. Srivastava, Structure of some classes of semisimple group algebras over finite fields, Bull. Korean Math.Soc. 351 (2014), no. 6, 1605-1614.

[18] _ The unit group of some special semi-simple group algebras, Quaestiones Mathematicae 39 (2016), no. 1.

[19] C. Musili, Representations of finite groups, Texts and Readings in Mathematics, vol. 3, Hindustan Book Agency, New Delhi, 2011, Reprint of the 1993 original.

[20] A. Olivieri and Á. del Río, An algorithm to compute the primitive central idempotents and the Wedderburn decomposition of a rational group algebra, J. Symbolic Comput. 35 (2003), no. 6, 673-687.

[21] A. Olivieri, Á. del Río, and J. J. Simón, On monomial characters and central idempotents of rational group algebras, Comm. Algebra 32 (2004), no. 4, 15311550.

[22] I. Van Gelder and G. Olteanu, Finite group algebras of nilpotent groups: a complete set of orthogonal primitive idempotents, Finite Fields Appl. 17 (2011), no. 2, 157-165. 